\title{
Intraoral Pressure Measurement during Mastication of Kelp
}

\author{
Kaoru KOHYAMA and Tomoko SAKAI \\ National Food Research Institute, 2-1-2 Kannondai, Tsukuba, Ibaraki 305-8642, Japan
}

Received April 12, 2000; Accepted October 4, 2000

\begin{abstract}
Masticatory pressure caused by chewing with molars was measured using a multiple-point sheet sensor. The sensor system is useful to directly indicate masticatory force and contact area with various subjects. Mastication of kelp snack, which is difficult to cut in several chewing strokes and thus seems to be a good food for masticatory training, was analyzed. Peak force, contact area at peak, peak pressure, duration, cycle time and impulse were highly varied among subjects, but were not influenced by the breaking force of the kelp. The experimental results show that humans did not change their masticatory pattern at least during the first several chews of the kelp samples which are difficult to cut with teeth. In the first chew, masticatory pressure is lower and duration and cycle time are longer than the following several chews. This suggests that subjects are afraid of an unknown sample texture. Individual peak force in mastication was independent of the contact area, but highly correlated with the peak pressure. Subjects with a high impulse value, corresponding to a large amount of work in mastication, had a high masticatory force, but did not show long duration.
\end{abstract}

Keywords: mastication, kelp, bite force, texture, multiple-point sheet sensor

Direct measurement of human mastication is a powerful technique to complement a mechanical test and sensory evaluation, and to relate them (Kohyama, 2000). This paper introduces a new technique to directly measure the masticatory pressure of humans by their molars.

Bite force has been measured with simple apparatuses, which are used mainly in a dental check-up. Since maximum bite force of adults is much higher than the force applied in chewing food (Bakke et al., 1989), sensors for dental check-up have a different pressure range from the optimum for masticatory force. Moreover, dental apparatuses are difficult to use in evaluating the mastication of food due to the rigidity and thickness of the sensing probe. Though micro pressure sensors attached to a tooth or denture (Anderson, 1953; Takahashi \& Nakazawa, 1987; Tornberg et al., 1988; Miwa, 1995) have shown valuable results, these are not applicable to many subjects (Kohyama, 2000). Meanwhile, some authors (Mioche et al., 1993; Peyron et al., 1994; Kohyama \& Nishi, 1997) applied small sensors to measure intra-oral force in biting various samples with the incisors. The role of molars in mastication is more important for food breakdown and texture perception. In this study, a multiple-point sheet sensor used in previous studies (Kohyama et al., 1997; Kohyama \& Nishi, 1997) is modified in order to measure the intra-oral force during the chewing of food with molars.

Lowering of the masticatory ability of youth, who may not be well trained in habitual food intake, has recently become a large problem in Japan (Kawamura \& Horio, 1989; Niki, 1991; Saito, 1992; Kishida, 1995). Kelp (Laminaria religiosa Miyabe) requires chewing many times until it can be swallowed, therefore it is a good food for masticatory training. In a preceding study which combined a mechanical test, sensory evaluation, electromyography and masticatory pressure measurement (Kohyama et

E-mail: kaoruk@nfri.affrc.go.jp al., 2000b), we reported that though the breaking force of samples differ, maximum force of the first chew was unchanged. Unlike other foods easily broken in one bite, a unique masticatory phenomenon observed within the first 5 chews of 4 kelp snacks is reported in this paper.

\section{Materials and Methods}

Samples The sample kelp was made and generously donated by Maejima Food Co. Ltd. (Kakogawa, Hyogo). Samples were cut to length of $15 \mathrm{~mm}$ in the fiber direction and 9.5 $\mathrm{mm}$ width, and classified into 4 groups by weight: I (mean weight $0.067 \mathrm{~g})$, II (0.091 g), III (0.113 g), and IV (0.152 g). The thickness, breaking force and sensory difficulty of mastication, and period of mastication increased in the order of $\mathrm{I}<\mathrm{II}<\mathrm{III}<\mathrm{IV}$, but breaking stress was similar among the samples as reported elsewhere (Kohyama et al., 2000b).

Masticatory pressure measurement Eleven healthy women (average age, 32.5 years) without functional problems in mastication voluntarily participated in this experiment.

Masticatory pressure was measured with an ISCAN $10 \times 10$ system (Nitta Corporation, Osaka). Measurement was done following the methods described in the previous reports (Kohyama et al., 1997; Kohyama \& Nishi, 1997), and only the sensor size was different. The sensor was composed of 100 sensing points made in 10 rows and 10 columns with a pitch of $1.27 \mathrm{~mm}$ printed on a flexible plastic film of $<0.1 \mathrm{~mm}$ thickness. Each subject used an individual sensor sheet, and each sensor was calibrated with a fixed load applied by an Instron apparatus (Model 6642) before the experiment.

A kelp sample was attached to the sheet sensor with adhesive tape. The fiber direction was adjusted parallel to the sensor column. Subjects inserted the sample on the sensor sheet between the upper and lower first molars of their habitual chewing side by themselves without touching the sample with their fingers. In a 


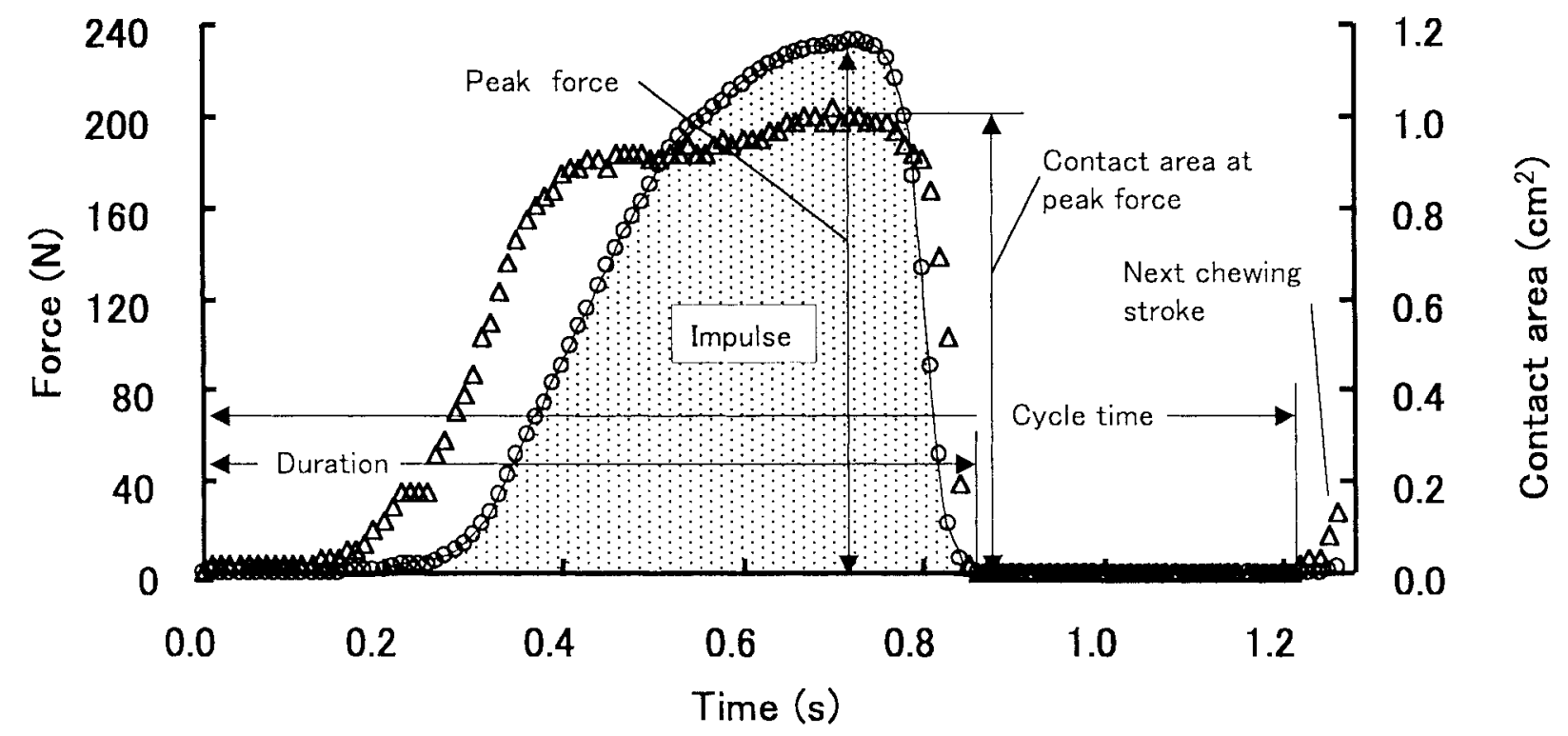

Fig. 1. Typical mastication curve of kelp. $\bigcirc$; sum of force detected by 80 sensing cells under the samples, and $\triangle$; contact area defined as sum of area of active sensing cells which detected non-zero pressure. Examples of parameters calculated (peak force, contact area at peak force, duration, cycle time, and impulse) are shown.

preliminary study, subjects did not change their chewing side during the first several chews of the kelp samples. Therefore, they normally chewed the sample with the sensor 5 times using their molars. Four samples were tested in random order, and each test was replicated 3 times.

Data analysis Masticatory pressure by 80 sensing cells ( 8 rows of 10 columns) under the kelp sample was analyzed. The masticatory force (i.e., sum of the force over the 80 cells) and contact area (sum of the cell area detecting non-zero pressure) were measured every $5 \mathrm{~ms}$. From the masticatory curves (Fig. 1 is an example), the peak pressure (the peak force divided by the contact area at the peak), duration, cycle time and impulse (area under the force-time curve) were calculated for the first 5 chews.

Statistical analyses were done using an SPSS package (ver.8.0J for Windows).

\section{Results and Discussion}

General masticatory behavior A masticatory curve is drawn by force and contact area versus time. As shown in Fig. 1, increase in contact area preceded increase in pressure during one chew. After the contact area reached to almost maximum, the pressure increased to the maximum and then both the area and pressure decreased very rapidly within $0.05 \mathrm{~s}$. Depending on the masticatory cycle, slope of the force-time curve varied among the subjects, but the force increase was much slower than the decrease in every case.

Figure 2 shows the pressure distribution on the kelp sample in Fig. 1. The anterior side of the subject is located at the left in the figure and the medial side appears on the front. As the subject chewed with her right side in this example, the outer (right) side of the dental arch comes at the back of the plot. Peak force is observed at $0.72 \mathrm{~s}$. As mentioned above, the rise in pressure took much longer than the reduction. It is evident that pressure is highly dependent on the positions due to the irregular shape of individual teeth. Two or three peaks corresponding to the outer and inner contact points of the upper and lower molars were observed. Subjects did not apply homogeneous pressure over samples during any of the chewing period, even though the clearance between the upper and lower teeth depressed to zero. In every case, there were inactive sensing cells (usually about $25 \%$ of the cells under the sample) that were not subjected to any pressure. Though kelp is fibrous, no differences between across and along fiber direction were observed. This is probably due to mastication with molars being close to simple compression that deforms independently in the fiber direction.

Differences among chewing cycles As shown in Fig. 3 and Table 1, the first chew was slightly different from the following several cycles. Lower peak pressures, longer duration and cycle time, and smaller impulse were observed than those in the following chewing cycles. The subjects know that kelp is tough food, but it is impossible to distinguish the toughness of each sample visually. Perhaps subjects were afraid of the unknown texture of the sample, and intended to investigate it during the first chew. Therefore, the first chew was weaker and a longer time was taken to determine the toughness. From the second chewing movement, since subjects knew the texture from the preceding chew, similar masticatory patterns are shown. This is because the kelp sample did not break or change very much with a small number of chews.

As previous authors reported (Anderson, 1953; Takahashi \& Nakazawa, 1987), masticatory force does not drastically change in a few chewing strokes. Kelp requires chewing many times before swallowing. The kelp was very thin (from $0.34 \mathrm{~mm}$ for sample I to $0.76 \mathrm{~mm}$ for IV) to attach the sensor sheet and fibrous and not easily cut; these properties made it possible to use a sheet sensor to measure masticatory force applied to the sample during several chews. Other intra-oral sensors fixed in or on teeth or prosthetics detect the force received by the teeth. Such sensors can show exactly what pressure the teeth received but cannot detect the pressure applied to the sample. In food mastication, 


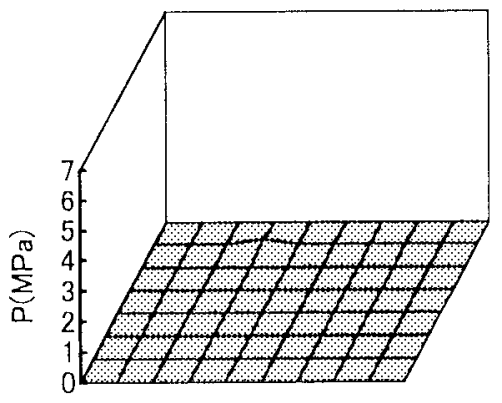

$0.1 \mathrm{~s}, 0.191 \mathrm{MPa}$

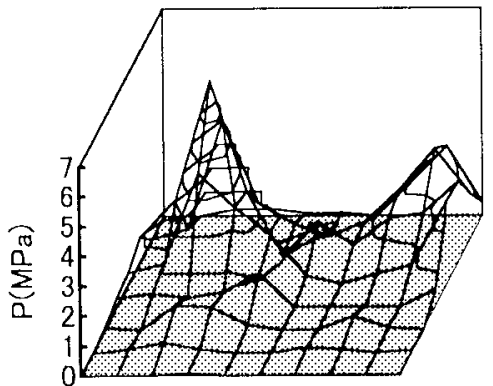

$0.4 \mathrm{~s}, 1.044 \mathrm{MPa}$

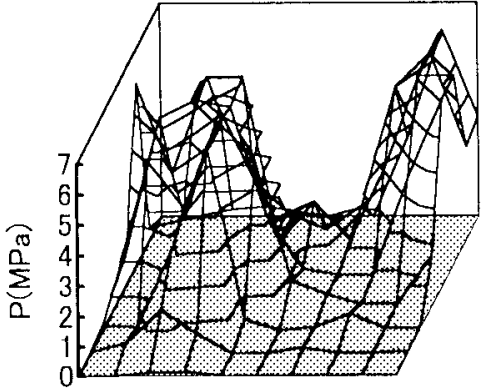

$0.7 \mathrm{~s}, 2.277 \mathrm{MPa}$

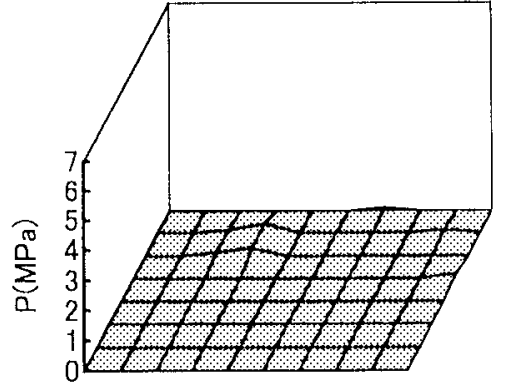

$0.2 \mathrm{~s}, 0.163 \mathrm{MPa}$

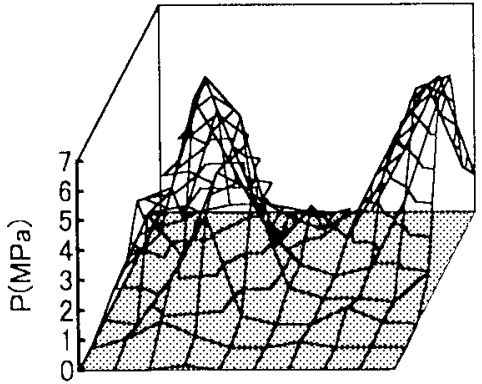

$0.5 \mathrm{~s}, 1.885 \mathrm{MPa}$

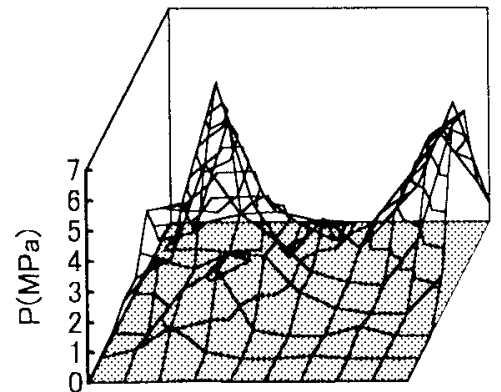

$0.8 s, 1.476 \mathrm{MPa}$

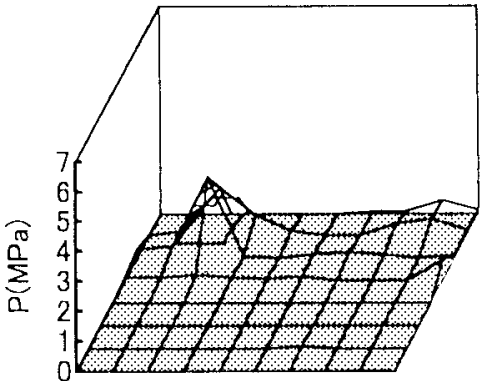

$0.3 \mathrm{~s}, 0.378 \mathrm{MPa}$

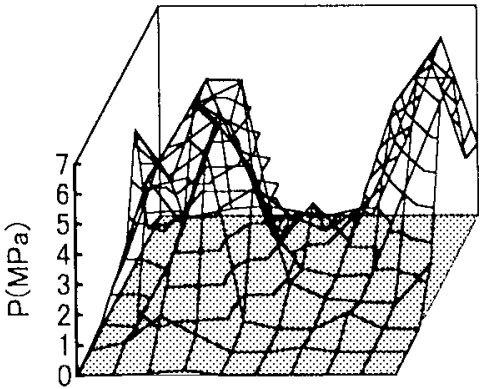

$0.6 \mathrm{~s}, 2.250 \mathrm{MPa}$

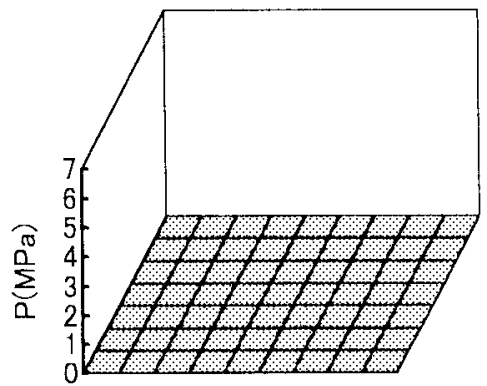

$0.9 \mathrm{~s}, 0.000 \mathrm{MPa}$

Fig. 2. An example of pressure distribution during the first chew of kelp. Frames every $0.1 \mathrm{~s}$ in the Fig. 1 . Time from the onset and active pressure (=masticatory force/contact area) are shown below each frame.

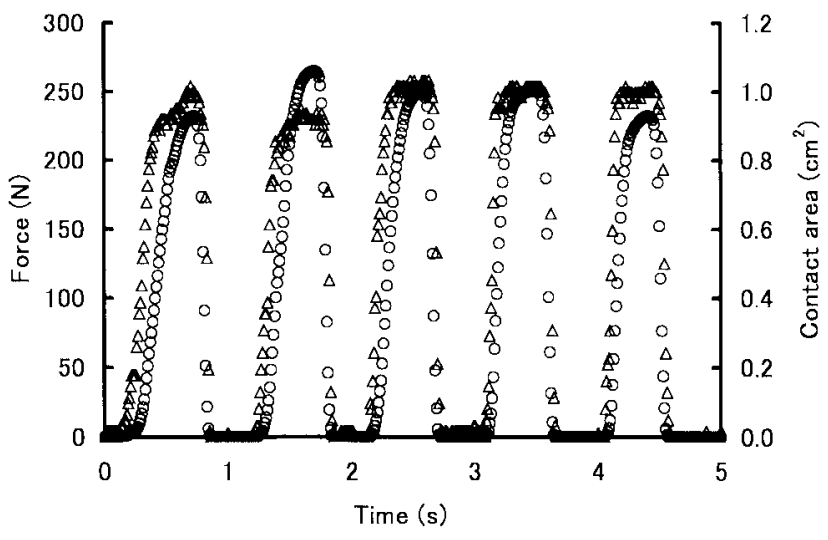

Fig. 3. Masticatory force and contact area during the first five chews. The first chew is also shown in Figs. 1 and 2.

humans do not usually use a single tooth, and sometimes change their chewing side. Contrary to the above mentioned merits, tolerance of the sheet sensor was found inadequate to detect pressure changes throughout the masticatory process with many chews. However, such a sensor was useful for the whole session of the first 5 chews in 12 trials ( 4 samples $\times 3$ replicates) without any damage.

Effects of samples Table 2 shows results of the analysis of variance among samples and parameters for 4 kelp samples at the first chew and the following 4 chews. Values at the first chew are different from those observed in the second to fifth chews, but a general tendency of sample dependence was commonly observed. Peak force, the contact area, chewing cycle, duration and impulse were independent of the sample used. Only the peak pressure showed significant difference $(p<0.05)$. The peak pressure for samples II and IV was lower than that for I and III, and the order corresponded neither to the breaking force in a tensile test nor sensory difficulty in mastication; that was in the order $\mathrm{I}<\mathrm{II}<\mathrm{III}<\mathrm{IV}$ (Kohyama et al. 2000b). The reason for this disagreement is unknown, but the observed significance level of the $t$-test was not so low (0.02 for first or 0.04 for the following 4 chews) as shown in Table 2 . We consider that difficulty in breaking kelp did not affect the masticatory pressure at least during the first several chews.

These results are similar to the observations of Peyron et al. 
Table 1. Comparison of masticatory pressure among the first 5 chews of kelp samples.

\begin{tabular}{|c|c|c|c|c|c|}
\hline Parameter & First chew & Second chew & Third chew & Fourth chew & Fifth chew \\
\hline Peak force $(\mathrm{N})$ & $102.06^{\mathrm{a}} \pm 4.25$ & $106.25^{\mathrm{a}} \pm 4.16$ & $107.61^{\mathrm{a}} \pm 4.15$ & $109.97^{\mathrm{a}} \pm 4.20$ & $109.90^{\mathrm{a}} \pm 4.02$ \\
\hline Contact area at peak $\left(\mathrm{cm}^{2}\right)$ & $0.757^{\mathrm{a}} \pm 0.014$ & $0.774^{\mathrm{a}} \pm 0.015$ & $0.773^{\mathrm{a}} \pm 0.016$ & $0.781^{\mathrm{a}} \pm 0.016$ & $0.789^{a} \pm 0.016$ \\
\hline Peak pressure $^{1}(\mathrm{MPa})$ & $1.381^{\mathrm{a}} \pm 0.046$ & $1.428^{\mathrm{ab}} \pm 0.047$ & $1.456^{\mathrm{ab}} \pm 0.047$ & $1.473^{\mathrm{b}} \pm 0.046$ & $1.454^{\mathrm{ab}} \pm 0.045$ \\
\hline Duration (s) & $0.595^{\mathrm{b}} \pm 0.018$ & $0.547^{\mathrm{a}} \pm 0.016$ & $0.544^{\mathrm{a}} \pm 0.015$ & $0.556^{\mathrm{a}} \pm 0.017$ & $0.554^{\mathrm{a}} \pm 0.018$ \\
\hline Cycle time (s) & $0.861^{\mathrm{b}} \pm 0.021$ & $0.830^{\mathrm{a}} \pm 0.018$ & $0.823^{\mathrm{a}} \pm 0.019$ & $0.841^{\mathrm{ab}} \pm 0.020$ & $0.843^{\mathrm{ab}} \pm 0.022$ \\
\hline Impulse $(\mathrm{N} \cdot \mathrm{s})$ & $30.388^{\mathrm{a}} \pm 1.575$ & $32.315^{\mathrm{ab}} \pm 1.617$ & $33.029^{\mathrm{ab}} \pm 1.709$ & $34.636^{\mathrm{b}} \pm 1.856$ & $35.388^{\mathrm{b}} \pm 1.908$ \\
\hline
\end{tabular}

Mean \pm standard error for 11 subjects $\times 4$ samples $\times 3$ replicates. Mean values followed by different alphabetical letter differ significantly $(p<0.05)$ among

chews determined by Tukey test. ${ }^{1}$ peak pressure=peak force/contact area.

Table 2. Parameters of masticatory pressure for different kelp samples.

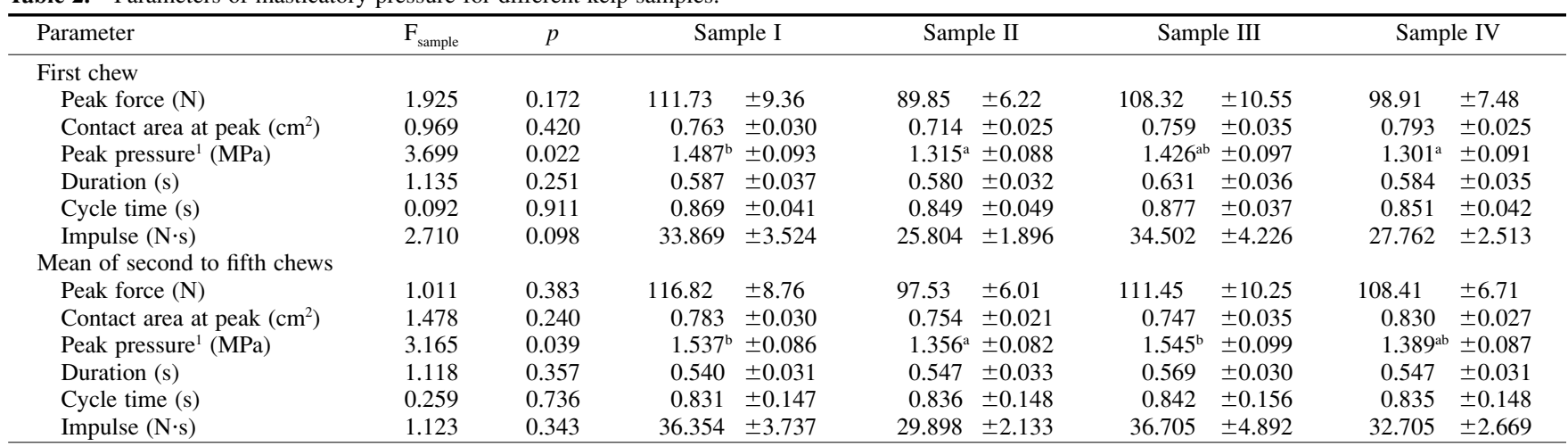

Mean \pm standard error for 11 subjects $\times 3$ replicates. Mean values followed by different alphabetical letter differ significantly $(p<0.05)$ between samples determined by paired-sample $t$-test. ${ }^{1}$ peak pressure=peak force/contact area.

Table 3. Individual parameter differences of masticatory pressure.

\begin{tabular}{lcc}
\hline Parameter & Mean & Minimum-maximum \\
\hline Peak force $(\mathrm{N})$ & 105.47 & $51.41-157.55$ \\
Contact area at peak $\left(\mathrm{cm}^{2}\right)$ & 0.771 & $0.595-0.922$ \\
Peak pressure $(\mathrm{MPa})$ & 1.419 & $0.799-2.167$ \\
Duration $(\mathrm{s})$ & 0.566 & $0.352-0.877$ \\
Cycle time $(\mathrm{s})$ & 0.848 & $0.535-1.210$ \\
Impulse $(\mathrm{N} \cdot \mathrm{s})$ & 32.733 & $15.364-71.0071$ \\
\hline
\end{tabular}

Mean values of 4 samples $\times 5$ chews $\times 3$ replicates.

(1994) that bite force remained constant for the soft silicone elastomers even though subjects sensed the difference in hardness. Variation in rheological properties among 4 kelp samples is smaller than that among the artificial silicone samples used by Peyron et al. (1994). Humans chew different kelp samples with a similar masticatory force.

Individual differences As commonly observed in masticatory parameters (Kohyama, 2000), differences among subjects $(p<0.0001)$ were larger in every parameter than that among samples. Table 3 shows mean, minimum and maximum values among 11 subjects; all of the parameters varied greatly. To characterize the individuals, correlation among the parameters is shown in Table 4. The peak pressure is defined as the peak force divided by the contact area at the peak, therefore, both the peak force and the contact area vary with subjects, and the peak force does not always correlate with the peak pressure. However, the results show very strong correlation between these two factors. This suggests that high masticatory force is not due to a large contact area at the peak but is related to high pressure. Duration and cycle time also had a high correlation coefficient. The im- pulse is the time-integral of force, high impulse value is affected by both the high peak force and long duration. However, the parameter is correlated only with the former.

Electromyography (EMG) is often used to analyze the masticatory phenomenon in humans (Kohyama, 2000). In most cases, activity in jaw-closing muscles is monitored. We previously tested EMG of the same kelp samples (Kohyama et al., 2000a; b). In the electromyograms of masticatory muscles, the duration and cycle time were not correlated, but maximum amplitude of muscle activity and time-integral of muscle activity were correlated $(p<0.01)$ (Kohyama et al., 2000a). The jaw-closing muscles also act to elevate the mandible the same as for mashing food, therefore low EMG activity is observed when a human does not produce any masticatory force. EMG signals appear during simple jaw closing without chewing food, while the sheet sensor did not detect masticatory pressure. The intraoral pressure sensor more directly measures the masticatory force than EMG. Noncorrelation between the duration and cycle time in the EMG measurement was probably because the duration included a simple jaw closing period before teeth touched sample food, and the value was the mean of muscle activity for both the chewing-side and the non-chewing side.

Though compression speed is variable during one chewing cycle in actual masticatory movement, the impulse is considered an analogue with the compression work in a mechanical test under a constant speed. This evidence suggests that the work required to mash food samples during mastication is mainly influenced by the masticatory force, and by the masticatory pressure correlated with the force. The above findings suggest that peak force observed during a chewing cycle may be the most important factor determining masticatory performance of an 
Table 4. Correlation coefficients among parameters of masticatory pressure.

\begin{tabular}{lcccc}
\hline & Contact area at peak & Peak pressure & Duration & Cycle time \\
\hline Peak force & 0.393 & $0.918^{a)}$ & -0.154 & -0.249 \\
Contact area at peak & & 0.021 & 0.190 & 0.160 \\
Peak pressure & & -0.281 & -0.352 & $0.440^{b)}$ \\
Duration & & $0.647^{c)}$ & 0.443 \\
Cycle time & & & 0.295 \\
\hline
\end{tabular}

Comparison of mean values of 4 samples $\times 5$ chews $\times 3$ replicates for 11 subjects. ${ }^{a)}, p<0.001 ;^{b}, p<0.01 ;{ }^{c}, p<0.05$.

individual.

Further scopes In the case of samples broken during the first bite, we obtained different evidence that the masticatory force and impulse are influenced by the texture of crackers (Kohyama \& Nishi, 1997). As brittle samples broke within 0.2$0.3 \mathrm{~s}$ of the first bite, mechanical properties of samples influenced the early stage of chewing. At around the peak in masticatory curves, a mixture of crushed food pieces and saliva are chewed, and the texture is not the same as the original one before mastication. A steep fall after rupture, zigzag or a smooth curve is observed within $0.3 \mathrm{~s}$ from the beginning of a bite. We speculate that independence of masticatory pressure on the sample hardness is observed in tough samples, whose breaking strain is high so that they are difficult to break in one chew. Experimental results of this study show that humans do not change their masticatory pattern, at least during the first several chews in the case of samples difficult to cut with the teeth like kelp. After completing the mastication with different masticatory period and chewing number, they distinguished the textural differences (Kohyama et al., 2000b), but it was difficult in the first few chews. We believe subjects did not recognize sample differences during the masticatory pressure measurement. When humans sense difficulty in mastication and whether or not they then change masticatory behavior will be studied in the future.

Acknowledgements This study was supported in part by Special Coordination Funds for Promoting Science and Technology of the Science and Technology Agency, and by a grant for Study of Physiological Functions of Food Components from the Ministry of Agriculture, Forestry and Fisheries of the Japanese Government.

\section{References}

Anderson, D.J. (1953). A method of recording masticatory loads. $J$. Dent. Res., 32, 785-789.

Bekke, M., Michler, L., Han, K. and Moller, E. (1989). Clinical significance of isometric bite force versus electrical activity in temporal and masseter muscles. Scand. J. Dent Res., 97, 539-551.
Kawamura, Y. and Horio, T. (1989). Effects of chewing exercise on the maximum biting force and chewing performance. Shika Kiso Igakukai Zasshi, 31, 281-290 (in Japanese).

Kishida, N. (1995). Masticatory functions and food habits. Nippon Soshaku Gakkai Zasshi, 5, 21-27 (in Japanese).

Kohyama, K. (2000). Studies of mastication and oral sensation, food texture. Nippon Shokuhin Kagaku Kogaku Kaishi, 47, 341-346 (in Japanese).

Kohyama, K. and Nishi, M. (1997). Direct measurement of biting pressures for crackers using a multiple-point sheet sensor. J. Texture Stud., 28, 605-617.

Kohyama, K., Nishi, M. and Suzuki, T. (1997). Measuring texture of crackers with a multiple-point sheet sensor. J. Food Sci., 62, 922 925.

Kohyama, K., Hatakeyama, E., Kobayashi, S., Seki. T., Takiguchi, T. and Suzuki, T. (2000a). Relationship between electromyograms of masticatory muscles for kelp and masticatory ability. Nippon Soshaku Gakkai Zasshi, 10, 41-49 (in Japanese).

Kohyama, K., Hatakeyama, E., Kobayashi, S., Yashiro. M., Azuma, T., Sakai, T. and Suzuki, T. (2000b) Masticatory difficulty and mechanical characteristics of kelp snacks. Nippon Shokuhin Kakagu Kogaku Kaishi, 47, 822-827 (in Japanese).

Mioche, L. Peyron, M.-A. and Auroy, P. (1993). The use of intraoral load cells in the study of texture perception. J. Texture Stud., 24, 361-373.

Miwa, Y. (1995). Three-dimensional chewing force during mastication of various foods. Shikaigaku, 58, 44-56 (in Japanese).

Niki, T. (1991). Mastication and health of children. Nippon Soshaku Gakkai Zasshi, 1, 11-18 (in Japanese).

Peyron, M.-A., Mioche, M. and Culioli, J. (1994). Bite force and sample deformation during hardness assessment of viscoelastic models of foods. J. Texture Stud., 24, 59-76.

Saito, S. (1992). Kamu no rokudan katsuyo (The six utilities of mastication). Hatsuratsu, 5, 3-9 (in Japanese).

Takahashi, J. and Nakazawa, F. (1987). Evaluation of food texture by masticatory pattern. 1. Analysis of chewing force pattern in mouth. Nippon Kasei Gakkaishi, 38, 107-113 (in Japanese).

Tornberg, E., Fjelkner-Modig, S., Ruderus, H., Glantz, P.O., Randow, K. and Stafford, D. (1985). Clinically recorded masticatory patterns as related to the sensory evaluation of meat and meat products. $J$. Food Sci., 50, 1059-1066. 